\title{
FACTORES ASOCIADOS CON EL DESEMPEÑO DEL PERSONAL QUE LABORA COMO OFICIALES DE TRÁNSITO Y SEGURIDAD EN LA UNIVERSIDAD DE COSTA RICA ${ }^{1}$
}

Ernestina Aguirre Vidaurre ${ }^{2}$ Giancarlo Guevara Francesa ${ }^{3}$

Institución: Universidad de Costa Rica

\section{COMO CITAR}

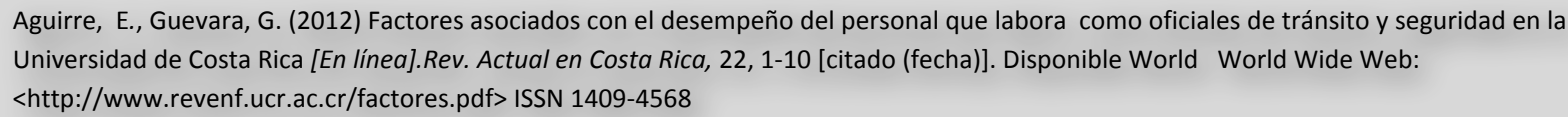

\section{RESUMEN}

El objetivo de este artículo es presentar los resultados de la investigación Factores que afectan el desempeño laboral de los y las oficiales de Tránsito y Seguridad, llevada a cabo en la Sede Ciudad Universitaria Rodrigo Facio de la Universidad de Costa Rica, durante el primer semestre del año 2009. La población estuvo conformada por 57 oficiales de tránsito y seguridad. Se aplicó un diseño cuantitativo de tipo descriptivo. Los instrumentos utilizados fueron la guía de observación y el cuestionario, los cuales fueron validados previamente. Los aspectos por analizar fueron las incapacidades, factores de riesgo que generan incapacitaciones, así como aquellos eventos que dañen el bienestar del trabajador; también, se emplearon resultados que proporcionó el Diagnóstico de Atención Primaria Ambiental (APA). Este estudio evidenció una alta incidencia de enfermedades crónicas que empeoran al exponerse a factores propios del trabajo, así como por la práctica de inadecuados estilos de vida como el tabaquismo, una mala alimentación y el sedentarismo. Del mismo modo, se presentan factores protectores como alto grado de satisfacción laboral, adecuadas relaciones interpersonales, conocimiento de los derechos y deberes laborales. Existen factores de riesgo como el grupo etáreo, jornadas laborales excesivas, horarios rotativos, así como alta movilidad por el campus. Como principal hallazgo se encontró que las causas más frecuentes de incapacidad laboral son las caídas y la gripe. Se concluye que los beneficios de un ambiente de trabajo saludable no se reducen al fortalecimiento de la salud del trabajador (a), sino a brindar un aporte positivo a la productividad e incrementar la motivación laboral, el espíritu de trabajo, la satisfacción en el trabajo y la calidad de vida general, aspectos que forman parte del objetivo de la práctica de la enfermera (o) en estos espacios.

Palabras clave: Salud-laboral, Factores-de-riesgo, Factores-protectores, Oficiales-de- seguridad, Tránsito.

\footnotetext{
${ }^{1}$ Fecha de Recepción: 23 de noviembre 2011

Fecha de aceptación: 15 de enero 2012

${ }^{2}$ Enfermera. Profesora asociada de la Universidad de Costa Rica. Máster en Administración Pública. Docente de la Escuela de Enfermería de la Universidad de Costa Rica. Investigadora del Programa de Investigación en Enfermería de la Universidad de Costa Rica. Miembro de la Comisión Técnica de Desarrollo del Recurso Humano en Salud, Ministerio de Salud de Costa Rica. Coordinadora de la Comisión del Programa Institucional de Lactancia Materna de la Universidad de Costa Rica. Correo electrónico: ernesagui@hotmail.com
}

${ }^{3}$ Enfermero. Labora en Coopesalud. Estudiante de la Maestría en Salud Pública. Correo electrónico: gian4418@hotmail.com 


\title{
FACTORS ASSOCIATED WITH THE PERFORMANCE OF OFFICIAL AS WORKS STAFF TRAFFIC AND SAFETY IN THE UNIVERSITY OF COSTA RICA ${ }^{1}$
}

Institution: University of Costa Rica

\author{
Ernestina Aguirre Vidaurre ${ }^{2}$ \\ Giancarlo Guevara Francesa ${ }^{3}$
}

CITED

Aguirre, E., Guevara, G. (2012). Factors associated with the performance of official as works staff traffic and safety in the university of Costa Rica.[En línea].Rev. Actual en Costa Rica, 22, 1-10 [citado (fecha)]. Disponible World World Wide Web: <http://www.revenf.ucr.ac.cr/factores.pdf> ISSN 1409-4568

\begin{abstract}
The objective of this article is to present the results of the investigation Factors affecting labor performance of the and the Traffic and Safety officers, held at Headquarters Rodrigo Facio University City University of Costa Rica during the first half of year 2009. The population consisted of 57 traffic officers and security. We performed a quantitative and descriptive. The instruments used were the observation guide and questionnaire, which were validated previously. The aspects to be analyzed were the incapacities, risk factors that generate incapacitations and events that harm the welfare of the worker also provided results that were used Diagnostic Environmental Care (PEC). This study showed a high incidence of chronic diseases that worsen when exposed to factors in work and the practice of inappropriate lifestyles such as smoking, poor diet and physical inactivity. Similarly, there are protective factors such as high job satisfaction, adequate interpersonal relationships, knowledge of labor rights and duties. Some risk factors such as age group, excessive working hours, rotating schedules, and high mobility around the campus. The main finding was found that the most frequent causes of work disability are falls and flu. It is concluded that the benefits of a healthy work environment are not limited to strengthening health worker (a), but to provide a positive contribution to productivity and enhance work motivation, the spirit of work, job satisfaction and overall quality of life, aspects that are part of the goal of nursing practice (o) in these spaces.
\end{abstract}

Keywords: Employment-disability, Risk-factors, Protective-factors, Transit-Security-Officers.

\footnotetext{
${ }^{1}$ Date of reception: November 23, 2011

Date of acceptance: January 15, 2012

${ }^{2}$ Enfermera. Enfermera. Profesora asociada de la Universidad de Costa Rica. Máster en Administración Pública. Docente de la Escuela de Enfermería de la Universidad de Costa Rica. Investigadora del Programa de Investigación en Enfermería de la Universidad de Costa Rica. Miembro de la Comisión Técnica de Desarrollo del Recurso Humano en Salud, Ministerio de Salud de Costa Rica. Coordinadora de la Comisión del Programa Institucional de Lactancia Materna de la Universidad de Costa Rica. Correo electrónico: ernesagui@hotmail.com
}

${ }_{3}^{3}$ Enfermero. Labora en Coopesalud. Estudiante de la Maestría en Salud Pública. Correo electrónico: gian4418@hotmail.com 


\section{INTRODUCCIÓN}

En Costa Rica, la Enfermería ha unido esfuerzos para promulgar un enfoque de salud integral, cuyo objetivo se basa en la preparación de profesionales capaces de trabajar en el desarrollo del cuidado personal y colectiva. Según lo muestra uno de los párrafos de la visión que tiene la Escuela de Enfermería de Universidad de Costa Rica (UCR), su fin último es promover la calidad de vida personal y de quienes están alrededor, de ahí que esta misión confiera un papel protagónico a las y los actores sociales (Escuela de Enfermería, 2009 s.p.)

Las líneas anteriores evidencian la función de la Enfermería contemporánea, la cual se enfoca en el cuidado de la persona, pero, más importante aún en el autocuidado que esa persona puede brindarse. La y el enfermero deben promover una participación activa en el proceso de salud, utilizando el pensamiento analítico, crítico e investigativo que se desarrolla a lo largo de toda la carrera.

Parra (2003) define Salud Laboral como aquella que "se preocupa de la búsqueda del máximo bienestar posible en el trabajo, tanto en la realización del trabajo como en las consecuencias de éste en todos los planos, físico, mental y social"' (p. 2)

Según Parra (2003), los riesgos para la salud relacionados con el trabajo son múltiples, sobre todo por la exposición a factores ambientales, motivo que insta a detectar tales necesidades en las poblaciones, de modo que reciban la atención debida. Tomando en cuenta lo expuesto y partiendo de que la salud involucra no solo un componente físico, sino uno psicológico y social, la Enfermería se inserta en el área laboral, con el fin de integrar estrategias que promuevan la salud en las personas trabajadoras de nuestra sociedad.

Por esta razón, la Escuela de Enfermería de la UCR desarrolla un plan de estudios con una visión integral de la salud de las personas; para ello, y para comprender mejor los procesos que viven, subdivide a la población en grupos etáreos y profundiza según su condición de salud o enfermedad. Dentro de éste plan se encuentra el curso Módulo de Adultez Sana, el cual permite a la población estudiantil desarrollar habilidades y destrezas en el trabajo con personas sanas, por tanto, el curso incluye objetivos concernientes al área hospitalaria de obstetricia y a la de salud laboral. En este último escenario es donde se desarrolla el presente estudio: pues se trabajó con el personal de la Sección de Tránsito y Seguridad de la Universidad de Costa Rica, quien se encarga de "brindar vigilancia y seguridad a los bienes de la Institución, salvaguardar la integridad física de las personas y regular y controlar lo relativo al tránsito en el campus universitario" (Valverde, 2001, p. 91)

Respecto del lugar de trabajo, la OPS, et al. (2000) considera que es

Un lugar prioritario para la promoción de la salud en el siglo XXI. La salud en el trabajo y los ambientes de trabajo saludables se cuentan entre los bienes más preciados de personas, comunidades y países. Un ambiente de trabajo saludable es esencial, no sólo para lograr la salud de los trabajadores, sino también para mejorar y mantener la productividad, la motivación laboral, el espíritu de trabajo, la satisfacción en el trabajo y la calidad de vida general. (p.4)

Los problemas en salud relacionados con las condiciones de trabajo generan un aumento de costos humanos y económicos que conllevan un importante problema de salud pública. 
Debido a lo anterior, el profesional en Enfermería cuenta con la capacidad para recurrir a una herramienta fundamental que le permite mejorar la salud laboral de los y las trabajadoras: la promoción de la salud. A partir de dicha perspectiva de la salud se identifican los determinantes sociales que definen la salud de una población, de modo que se puedan reforzar factores protectores, eliminar factores de riesgo y cumplir con el principal objetivo de estos profesionales de salud : mejorar la calidad de vida del ser humano.

\section{MATERIALES Y METODOS}

La población participante correspondió a 57 personas de la Sección de Seguridad y Tránsito, de las cuales 47 son del departamento de seguridad, 8, oficiales de tránsito y 2, personal de administración.

De acuerdo con Barrantes (2006), "la investigación corresponde a un diseño no experimental, cuantitativo, descriptivo" (p. 70) Se analizaron los factores que interfieren en el desempeño laboral de los y las oficiales de tránsito y seguridad de la Ciudad Universitaria Rodrigo Facio; como se muestra en el problema de investigación. El universo de estudio que se analizó se encuentra constituido por las y los Oficiales de Seguridad y Tránsito, compuesto por 130 personas. Cabe destacar que no se tomó la totalidad de la población, sino que se extrajo una muestra probabilística formada por 57 personas de las cuales 47 son del departamento de seguridad, 8 son oficiales de tránsito y 2 pertenecen al área administrativa. Esta muestra fue tomada empleando el método de muestreo aleatorio simple.

Para identificar las potenciales causas generadoras de incapacidades dentro de esta población, se evaluó el estado físico y generalidades de salud mental de la población; también, se aplicó un cuestionario para la recolección de datos, el cual fue previamente validado.

La entrevista constó de ocho preguntas personales que incluyeron aspectos relacionados con la situación laboral de los y las oficiales, así como información respecto de las incapacidades y factores de riesgo que originan las incapacitaciones, así como la aparición de algún evento que altere el bienestar del trabajador.

Además, se utilizaron los resultados del Diagnóstico de Atención Primaria Ambiental (APA) elaborado por los investigadores y la población participante; como se mencionó, el APA es una estrategia dirigida a proteger y mejorar la salud de la población y el ambiente, creando un medio sano mediante la promoción y ejecución de acciones básicas y preventivas en el nivel local, propiciando relaciones entre sectores y diversas disciplinas, así como con la comunidad.

Este diagnóstico fue elaborado previo a la investigación y se utilizaron los datos recolectados a través de cuestionarios autoaduministrados a los y las oficiales. El diagnóstico permitió, en una primera instancia, conocer las condiciones ambientales en las cuales se desenvuelven los y las oficiales y la viabilidad del trabajo; además, permitió conocer los horarios de trabajo, las funciones, sus conocimientos en materia legal-laboral e, inclusive, las relaciones interpersonales, familiares y laborales.

Tras el análisis de los factores de riesgo y factores protectores identificados, se logró establecer diagnósticos y generar acciones para minimizar el impacto que el trabajo puede ocasionar en los trabajadores de manera que mantengan su calidad de vida. 
Para su implementación, se utilizando una metodología de participación activa entre facilitadores (estudiantes de Enfermería) y los participantes, la cual intensificó la recepción del mensaje y un aprendizaje para ambas partes (estudiantes y oficiales)

\section{Consideraciones éticas}

Para garantizar la confidencialidad de los datos suministrados por cada una de las personas que formaron parte de este estudio, previamente, se les informó acerca del contenido de la entrevista, así como del uso que se le daría y, como medida de reafirmación, esta información se incluyó por escrito en cada instrumento.

Además, las investigadoras se aseguraron de no utilizar nombres, iniciales o números con los que se pudiese identificar a los participantes del estudio.

\section{RESULTADOS}

Según la entrevista aplicada a 57 oficiales de Tránsito y Seguridad de la Universidad de Costa Rica, Sede Central Rodrigo Facio, de la población un 84\% labora como oficiales de Seguridad y el restante 16\% pertenece al Área de Tránsito. De esta muestra, la mayoría de oficiales tenían menos de 4 años de laborar, cifra que representa un $32 \%$ de la muestra participante, seguido por un $24 \%$ de los oficiales que posee entre 5 y 9 años. Existe un $12 \%$ que se ubica entre los 20 y 24 años de laborar. Por otra parte, la mayoría de los y las oficiales (un 84\%) trabaja doble turno.

Se determinó que el $88 \%$ de los oficiales se ha incapacitado alguna vez y solo un $12 \%$ no la ha hecho.

Las principales causas de incapacidad corresponden a gripes y caídas. Luego, en cuanto a importancia, siguen las incapacidades provocadas por agentes externos como accidentes vehiculares o caídas, pero hay una alta incidencia de enfermedades crónicas que empeoran con la exposición a factores propios del trabajo e inadecuados estilos de vida entre los que figuran el tabaquismo, una mala alimentación y el sedentarismo.

El estrés, según se evidencia a partir de las entrevistas, es un factor que aparece en prácticamente todos los oficiales, el cual se debe a la responsabilidad que implica el resguardo de los estudiantes y de la institución; sin embargo, aunque no es una causa de incapacidad, sí provoca problemas como gastritis, insomnio, inadecuadas relaciones interpersonales, cefaleas e inadecuadas prácticas alimenticias, por lo tanto, urge tratar el estrés y proporcionar técnicas para manejarlo.

En cuanto a la categoría "otras causas de incapacidad", un 37\% de la población mencionó enfermedades crónicas como cáncer, asma, diabetes, osteocondriasis, síndrome nefrótico, alergias y procesos postoperatorios.

En cuanto a la duración de estas incapacidades, un 30\% se extiende entre 1 y 5 días; un 35\%; entre 6 y 20 días y solo un $10 \%$ de la muestra se ha incapacitado por más de un año.

La mayoría de oficiales concuerdan en que el campus presenta múltiples riesgos para su salud integral y que dichos factores van a depender de las funciones que cumplen los oficiales en su trabajo. Algunos de los 
riesgos mencionados son exceso de humedad, ruido, humo, gases, polvo, respecto de los que un $80 \%$ los considera un factor de riesgo para su salud.

Igualmente, se destacan zonas de mayor riesgo, como la Caseta de Derecho y el LANANME, ya que ambas zonas constituyen las principales entradas de vehículos a la Universidad de Costa Rica, por lo que los oficiales que se encuentran supervisando la entrada y salida de vehículos y están altamente expuestos a los gases y residuos de los automóviles. Además, existen otros factores que los afectan, tales como el estrés que genera estar en contacto directo con personas que podrían reaccionar inadecuadamente al no encontrar parqueo, la contaminación sónica, entre otros.

No obstante, se identificaron otros factores positivos que potencian la calidad de vida de estos trabajadores, por ejemplo, el 98\% de esta población refleja tener un alto grado de satisfacción respecto de la funciones asignadas y al trabajo realizado. Además, un 92\% refiere mantener adecuadas relaciones interpersonales entre compañeros de trabajo y con sus superiores y un $83 \%$ de estos trabajadores posee conocimiento acerca de los derechos y garantías laborales.

\section{DISCUSIÓN}

El grupo de edad predominante oscila entre 36 y 59 años, lo que corresponde a la etapa de adultez intermedia, etapa cuya característica es que "existe el riesgo a un mayor deterioro y desgaste de la salud de las personas" (Papalia, 2004, p.23), por tanto, su edad los hace más vulnerables a sufrir enfermedades y lesiones relacionadas con su ambiente de trabajo en comparación con el personal joven.

Al analizar las causas de las incapacitaciones, se identificaron en su mayoría cuadros gripales, los cuales se pueden asociar, según Parra (2003), a "enfermedades comunes" que "muchas veces tienen una causa directa en el trabajo, cuestión que si se analiza con precisión, puede aplicarse a la gran mayoría de patologías en el adulto que reconocen factores ambientales". (p.8). Este punto es rescatable considerando la gran exposición que los oficiales tienen a factores ambientales como condiciones climáticas, vibraciones, ruido excesivo, malas posturas, contaminación ambiental, humo, gases, polvo, agentes químicos o vapores contaminantes que pueden alterar la salud de este personal. Al respecto, Parra (2003) considera que

...en todo lugar de trabajo existe un ambiente físico que rodea a las personas trabajando. Entre el ambiente y las personas se produce una interacción que puede causar daño si se sobrepasan determinados niveles de equilibrio normal. Los procesos de trabajo, en general, además producen una modificación del ambiente, muchas veces aumentando factores de riesgo. ( $\mathrm{p} 23$ )

Otro agente que promueve las incapacidades corresponde a las caídas, relacionadas en su mayoría con la movilidad del personal alrededor de todo el campus. Según Parra (2003), "los pisos húmedos, resbalosos y/o en mal estado, mala iluminación, ausencia de normas de trabajo seguro, falta de elementos de protección personal, los vehículos y la maquinaria en mal estado, son factores de riesgo que generan gran cantidad de accidente."(p.7) Por esta razón es necesario evaluar las condiciones de los zapatos que utilizan para determinar si son las óptimas para el cargo que desempeñan. 
En cuanto a los accidentes vehiculares, hay una alta incidencia de accidentes en motocicletas en comparación con los accidentes en patrullas; en relación con lo anterior, Polidoro (sf) menciona que "este tipo de accidentes son la parte más visible del daño corporal. Se calcula que los accidentes representan solamente una cuarta parte de la mortalidad derivada del trabajo" (sp). Estos problemas están relacionados con las incapacidades parciales permanentes que provocan una disminución de facultades o aptitudes para el trabajo.

Otro punto importante está ligado a los problemas ergonómicos, asociados en su mayoría con posturas fijas e incómodas (estar de pie o sentado por periodos largos y con inadecuadas posturas), de lo cual puede colegirse un mal uso de los principios de la mecánica corporal, tal como lo menciona Parra(2003), quien menciona que "los problemas aparecen cuando se le exige a las personas que permanezcan en una misma postura durante un tiempo excesivo, en malas posturas o que realicen movimientos y fuerzas más allá de sus capacidades". (p. 20)

Aunado a ello, el peso del equipo (aproximadamente $3.5 \mathrm{~kg}$ ), provoca molestias lumbares. Es importante recalcar que la inadecuada mecánica corporal en el momento de manipular objetos pesados puede desencadenar problemas músculo-esqueléticos además de la formación de hernias, otra de las causas de incapacidad.

Por otra parte, entre los principales problemas gastrointestinales, en primer lugar, podemos mencionar la diarrea y luego, la gastritis. Según la teoría consultada, las diarreas presentan una mayor incidencia durante la época de invierno, debido a inadecuados hábitos de higiene y desórdenes alimenticios. De igual forma, podemos asociar los desórdenes alimenticios con la aparición de gastritis, la cual es una manifestación fisiológica del estrés. Parra (2003) dice que "de mediano a largo plazo, los trabajadores en turno tienen un mayor riesgo de trastornos digestivos. Ya que no se produce una adaptación en los trabajadores nocturnos permanentes, porque no hay una transformación en seres humanos nocturnos". (p. 17)

Entre las incapacidades relacionadas con "otras causas", estas se asocian con enfermedades crónicas, las cuales pueden empeorar con la exposición a factores propios del trabajo e inadecuados estilos de vida como lo es el tabaquismo, una mala alimentación y el sedentarismo que, por sí, pueden activar la enfermedad, máxime si se añaden "los factores ambientales del trabajo como los turnos de noche, la jornada extensa o el exceso de calor o de frío pueden ser agravantes del problema”. (Parra, 2003, p. 8)

Aun cuando el estrés es un factor presente en prácticamente todos los oficiales, muy pocos se ven obligados a incapacitarse por esta razón; la responsabilidad del resguardo de los estudiantes y de la institución en general representa uno de los principales motivos de este padecimiento que va a somatizarse en forma de problemas como gastritis, insomnio, inadecuadas relaciones interpersonales, entre otros. Otra consecuencia que está relacionada con el estrés son las migrañas que además del estrés se pueden asociar a deficientes prácticas alimenticias, condiciones del ambiente físico como ruido, luz y calor entre otros, lo cual concuerda con la opinión de (Polidoro., s.f.) quien afirma que "una persona experimenta estrés cuando percibe un desequilibrio entre las demandas que tiene planteadas y la capacidad para responder a las mismas, en condiciones en las que fallar puede tener importantes consecuencias negativas" (s.p.)

Por lo anterior, se consideró que el horario nocturno puede generar cansancio, debilidad y estrés producto de la alteración del patrón del sueño. Según la OPS (2005) “el trabajo nocturno es particularmente fatigoso porque 
significa trabajar en las horas de reposo fisiológico y dormir durante el día, lo que resulta ser menos recuperador que el sueño en horas de la noche" (s.p.)

Sumado a los horarios nocturnos, se encontró que la mayoría realiza doble turno (el $84 \%$ de la muestra). Al respecto, la OPS (2005) considera que

...los servicios que solicitan continuamente al personal que trabaje horas extras, o que esté disponible durante más de 24 horas, o que trabaje turnos intercambiados a la semana sin tiempo de recuperación adecuada, están utilizando las prácticas de trabajo que constituyen un riesgo de seguridad y salud ocupacional ". (s.p.)

También, es importante tomar en cuenta que el estudio reveló aspectos positivos, ya que se puede apreciar que la gran mayoría del personal de seguridad y tránsito cataloga de buena a excelente la relación con sus compañeros de trabajo y con sus superiores, lo cual se considera un factor protector en esta población, debido a que genera un adecuado ambiente laboral que fomenta el apoyo, la cooperación y la comprensión entre compañeros de trabajo y superiores. En relación con lo anterior, Parra (2003) afirma que

La comunicación también es un aspecto esencial de la convivencia humana y, en ese sentido, el espacio de trabajo es un lugar de convivencia entre personas, donde se crean redes de apoyo, amistades, se forman y fortalecen familias, entre otras importantes consecuencias de este carácter social del trabajo. Por lo mismo, favorecer la comunicación en el trabajo mejora la satisfacción (p. 24)

La información mencionada se relaciona con otro de los aspectos ligados al grado de satisfacción de los participantes en cuanto al desarrollo de sus funciones, respecto de lo que Robbins (citado por Caballero 2002) indica que "una persona con un alto nivel de satisfacción en el puesto tiene actitudes positivas hacia el mismo; una persona que está insatisfecha con su puesto tiene actitudes negativas hacia él" (p.2); tal idea es corroborada en los resultados, ya que un $98 \%$ de los trabajadores de seguridad y tránsito siente un alto grado de satisfacción en sus labores por lo que se asegura un ambiente laboral adecuado y, por ende, el buen desempeño de sus funciones.

Dada la exposición del personal a muchos elementos que ponen en riesgo su salud, es necesario que utilicen equipo especializado que les permita minimizar estos riesgos (el casco de seguridad, el chaleco protector, las botas, los guantes y el equipo de defensa personal, dígase armas, esposas y batón policial). En cuanto a este punto, Parra (2003) menciona que

La seguridad implica el uso de técnicas que permitan eliminar o reducir el riesgo de sufrir lesiones en forma individual o daños materiales en equipos, máquinas y herramientas (...) desde el punto de vista de la seguridad es de mucha utilidad considerar estos mecanismos para adoptar medidas preventiva (p. 11)

A pesar de que este equipo representa un factor de protección para los oficiales, también puede generar problemas musculoesqueléticos como se mencionó; razón por la que es necesario evaluar la condición física de los trabajadores rutinariamente. 
Como último aspecto, la gran mayoría del personal conoce sus derechos y garantías laborales, una herramienta más de protección a la hora de cumplir con sus funciones. Al respecto, la Organización Internacional del Trabajo (1992) afirma que

...las autoridades competentes, previa evaluación de los riesgos para la seguridad y la salud y previa consulta con las organizaciones más representativas de empleadores y de trabajadores, deberían adoptar y mantener en vigor leyes o reglamentos nacionales que aseguren la seguridad y la salud de los trabajadores en el trabajo (...) y que protejan a las personas que se encuentren en sus inmediaciones de todos los riesgos que pueden derivarse del su trabajo (s.p.)

\section{CONCLUSIONES}

Los beneficios de un ambiente de trabajo saludable no se reducen al fortalecimiento de la salud del trabajador y la trabajadora, sino a brindar un aporte positivo a la productividad e incrementar la motivación laboral, el espíritu de trabajo, la satisfacción en el trabajo y la calidad de vida general, aspectos que forman parte del objetivo de la práctica de la enfermera (o) en estos espacios.

El lugar de trabajo saludable no siempre es una realidad a pesar de los intentos que se han tratado de implementar en la Universidad de Costa Rica, ya que existen variables no modificables que afectan a los y las trabajadoras.

\section{REFERENCIAS}

Barrantes, E. R. (2006) Investigación: un camino al conocimiento, un enfoque cualitativo y cuantitativo. San José, Costa Rica.: EUNED.

Caballero, K (2002), El concepto de "satisfacción en el trabajo" y su proyección en la enseñanza. Universidad de Granada. Recuperado el 19 de junio de 2009 de: http://www.ugr.es/ recfpro/rev61COL5.pdf.

Escuela de Enfermería, Universidad de Costa Rica (2009). Visión Escuela de Enfermería. Recuperado el 20 de marzo del 2009 de http://www.enfermeria.ucr.ac.cr/vision.htm

OPS, OMS, DSA y PRST (2000). Estrategias de la promoción de la salud en los lugares de trabajo de América Latina y el Caribe. Recuperado el 17 de abril del 2009

http://www.who.int/occupational_health/regions/en/oehpromocionsalud.pdf

Organización Internacional del Trabajo (1992), Seguridad y salud en la construcción. Repertorio de recomendaciones prácticas de la Ginebra, Oficina Internacional del Trabajo ( ). Recuperado el día 19 junio de $2009 \mathrm{de}$ http://www.ilo.org/public/english/protection/safework/cops/spanish/download/s920894.pdf

Organización Panamericana de la Salud (2005). Salud y Seguridad de los trabajadores del sector salud. Recopilación de lecturas Promoción de la Salud, Escuela de Enfermería, UCR, 2009. 


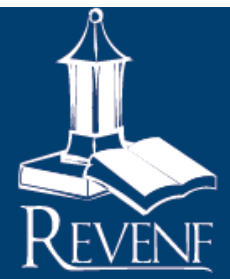

Papalia, E. Wendkos, O \&Duskin, R. (2004). Desarrollo humano. Edad adulta temprana. Parte seis, capítulos 13 y 14 y Edad adulta intermedia. Parte siete, capítulos 15 y 16. Novena Ed. México: McGraw Hill.

Parra, M. (2003). Conceptos básicos de Salud Laboral. Recuperado el 17 de abril del 2009 de http://www.oitchile.cl/pdf/publicaciones/ser/ser009.pdf

Polidoro, E. (S.F.) Salud laboral y prevención de riesgos laborales, aproximación desde una perspectiva de género. Recuperado el 17 de abril del 2009 de http://www.emakunde.es/images/upload/relaciones_1_c.pdf

Valverde, G. (2001). Entorno Operativo y Legal del Sistema de Seguridad de la Universidad de Costa Rica: Una propuesta de Mejora. Tesis de Maestría, Escuela de Administración de Empresas con énfasis, Universidad de Costa Rica. 